\title{
Flatness and structural analysis as a constructive framework for private communication
}

\author{
B. Dravie ${ }^{\mathrm{a}}$, P. Guillot ${ }^{\mathrm{b}}$, G. Millérioux ${ }^{\mathrm{a}}$ \\ ${ }^{a}$ Université de Lorraine, CRAN, UMR 7039, France, \\ CNRS, CRAN, UMR 7039, France \\ (e-mail: \{brandon.dravie,gilles.millerioux\}@univ-lorraine.fr). \\ ${ }^{b}$ Université Paris 8, LAGA, France, \\ (e-mail: philippe.guillot@univ-paris8.fr)
}

\begin{abstract}
This paper deals with the use of control theoretical concepts, essentially flatness, along with structural analysis, in the context of private communication. A new and systematic methodology to design a cryptographic architecture belonging to the special class of ciphers called Self Synchronizing Stream Ciphers (SSSC) is proposed. Till now, the constructions of SSSC were based on automata with finite input memory involving shifts or triangular functions ( $T$-functions) as state transition functions. Besides, only ad-hoc approaches were available in the literature. The contribution of this paper is to propose a general framework to design SSSC involving dynamical systems taking the form of switched linear systems over finite fields with arbitrary state transition functions. An example of cipher with complete specifications is given. Its implementation on a small scale breeding of an ICS-SCADA equipment is described.
\end{abstract}

Keywords: flatness, LPV and switched linear systems, structural analysis, ciphers

\section{Introduction}

Synchronization has been an important topic in automatic control for years. Roughly speaking, by synchronization, it is meant correlated (according to given criteria) behaviors of at least two or more interconnected entities in virtual or physical networks. Throughout the past centuries, scientists have attempted to explain the emergence of order through the concept of synchronization. C. Huygens in 1665 can be considered as a pioneer. A lot of examples of synchronized phenomena (see [26]) are borrowed from nature, biology, neuroscience, physiology and more recently social networks.

Synchronization can be a very efficient way of tackling engineering issues as well. For example, there is a growing interest in cooperative control problems. Such problems involve several autonomous entities (also called agents) 
which try to collectively reach a global objective by a suitable connectivity or by adequate couplings. The related applications are mobile robots, unmanned and autonomous vehicles, satellites, air traffic control. Synchronization is also central in communication: video broadcasting, Phase Lock Loop-based equipment. When synchronization must occur in a peer-to-peer communication setup without any external control, it is called self-synchronization. It turns out that self-synchronization is central in cryptography, more specifically, in symmetric cryptography involving the so-called Self-Synchronizing Stream Ciphers (SSSC) (see [20]). Such ciphers are based on generators which can take the form of dynamical systems operating on finite fields and must deliver sequences of high complexity. Those sequences are used to scramble the information to be safely transmitted. For proper decryption, those sequences must be self-synchronized.

Self-Synchronizing Stream Ciphers were patented in 1946. The self-synchronization property has many advantages and is especially relevant to group communications. Since 1960, specific SSSC have been designed and are still used to provide bulk encryption (for hertzian line, RNIS link, ...) in military applications or governmental radio mobile networks. In the early 90 s, studies have been performed in $[19,12]$ to propose secure designs of SSSC. These works have been followed by effective constructions $([12,24,11])$, but till now, all of these SSSC have been broken, which motivates the search of new constructions of SSSC. It is this issue that is investigated in this paper.

More precisely, we aim at addressing the design of SSSC in terms of control theoretical concepts. The ciphers being viewed as dynamical systems, the main result is that the concepts of flatness together with structural analysis and graph-theory allow for a convenient and systematic way to construct classes of SSSC which are more general than the ones proposed so far. Indeed, it will be shown that the resulting ciphers can incorporate dynamical systems with state transition functions more general than the so-called $T$-functions which are known to suffer from weakness [22]. Hence, we can expect that this generalization yields more secure SSSC. The special class of flat Linear Parameter Varying (LPV) systems is motivated. Let us stress that since the dynamics operate on finite fields, all the variables, including the varying parameters, take value in a finite set of elements. Hence, the LPV system can be considered as a switched linear system with a number of modes depending on the cardinality of this set.

The paper is organized as follows. Section 2 is devoted to the problem statement. The architecture of Self-Synchronizing Stream Ciphers is presented in terms of dynamical systems. In Section 3, the special class of LPV dynamical systems is presented, the definition of difference flatness is introduced and is particularized to this class. An algebraic characterization in terms of state space matrix representation is provided. The connection between flatness and SSSC is made. It is shown that an automaton with a finite input memory, as required for an SSSC, can be systematically obtained from the left inverse of a flat system. In Section 4, a construction of a family of SSSC involving LPV 
automata is provided. It is based on structural analysis and graphs. A simple example of construction is given to make a clear understanding of the method. Then, the design of a realistic example and its implementation on a small scale breeding of an ICS-SCADA equipment are described.

\section{Self-Synchronizing Stream Ciphers and dynamical systems}

\subsection{Generalities on stream ciphers}

For a stream cipher, it must be given an alphabet $A$, that is, a finite set of basic elements named symbols. Hereafter, the index $k$ will stand for the discrete-time. On the transmitter part, the plaintext (also called information or message) $m \in \mathcal{M}$ ( $\mathcal{M}$ is the message space) is a string of plaintext symbols $m_{k} \in A$. Each plaintext symbol is encrypted, by means of an encryption (or ciphering) function $e$, according to:

$$
c_{k+b}=e\left(z_{k+b}, m_{k}\right)
$$

where $z_{k} \in A$ is a so-called keystream (or running key) symbol delivered by a keystream generator. The function $e$ is invertible for any prescribed $z_{k}$. The resulting quantity $c_{k} \in A$ is the ciphertext symbol. The integer $b \geq 0$ stands for a potential delay between the plaintext $m_{k}$ and the corresponding ciphertext $c_{k+b}$. This is explained by computational or implementation reasons, for instance pipelining (see [8] for example). Consequently, for stream ciphers, the way how to encrypt each plaintext symbol changes on each iteration. The resulting ciphertext $c \in \mathcal{C}$ ( $\mathcal{C}$ is called the ciphertext space), that is the string of symbols $c_{k}$, is conveyed to the receiver through a public channel.

At the receiver side, the ciphertext $c_{k}$ is decrypted according to a decryption function $d$ which depends on a running key $\widehat{z}_{k} \in A$ delivered, similarly to the cipher part, by a keystream generator. The decryption function $d$ obeys the following rule. For any two keystream symbols $\widehat{z}_{k+b}, z_{k+b} \in A$, it holds that

$$
\widehat{m}_{k+b}:=d\left(c_{k+b}, \widehat{z}_{k+b}\right)=m_{k} \text { whenever } \widehat{z}_{k+b}=z_{k+b} .
$$

Equation (2) means that the running keys $z_{k}$ and $\widehat{z}_{k}$ must be synchronized for a proper decryption. The generators delivering the keystreams are parametrized by a secret key denoted in the sequel by $\theta \in \mathcal{K}(\mathcal{K}$ is the secret key space). The distinct classes of stream ciphers (synchronous or self-synchronizing) differ each other by the way on how the keystreams are generated and synchronized. Next, we detail the special class of stream ciphers called Self-Synchronizing Stream Ciphers.

\subsection{Keystream generators for Self-Synchronizing Stream Ciphers}

A well-admitted approach to generate keystreams has been first suggested in [19]. It is based on the use of so-called state automata with finite input memory 
as described below. This is typically the case in the cipher Moustique [18]. At the ciphering side, the automaton delivering the keystream takes the form:

$$
\left\{\begin{array}{l}
q_{k+1}=g_{\theta}\left(q_{k}, c_{k+b}\right), \\
z_{k+b}=h_{\theta}\left(q_{k}\right)
\end{array}\right.
$$

where $q_{k} \in A$ is the internal state, $g$ is the next-state transition function parametrized by $\theta \in \mathcal{K}$. As previously stressed, the delay $b$ is due to the fact that the output (also called filtering) function $h$ is pipelined with an architecture involving $b$ stages. If such an automaton has finite input memory, it means that, by iterating (3) a finite number of times, there exists a function $\ell_{\theta}$ and a finite integer $M$ such that

$$
q_{k}=\ell_{\theta}\left(c_{k+b-1}, \ldots, c_{k+b-M}\right),
$$

and thus,

$$
z_{k+b}=h_{\theta}\left(\ell_{\theta}\left(c_{k+b-1}, \ldots, c_{k+b-M}\right)\right) .
$$

Actually, the fact that the keystream symbol can be written in the general form

$$
z_{k+b}=f_{\theta}\left(c_{k-\ell}, \ldots, c_{k-\ell^{\prime}}\right)
$$

with $f_{\theta}$ a function involving a finite number of past ciphertexts from time $k-\ell$ to $k-\ell^{\prime}\left(\ell, \ell^{\prime} \in \mathbb{Z}\right)$, is a common feature of the SSSC. Equation (6) is called the canonical equation.

Remark 1. The outcome of implementing the recursive form (3) instead of directly implementing the canonical form (6) is that we can obtain nonlinear functions $f_{\theta}$ of high complexity by implementing simpler nonlinear functions $g_{\theta}$. The complexity results from the successive iterations which act as composition operations.

At the deciphering side, the automaton takes the form

$$
\left\{\begin{array}{l}
\widehat{q}_{k+1}=g_{\theta}\left(\widehat{q}_{k}, c_{k+b}\right) \\
\widehat{z}_{k+b}=h_{\theta}\left(\widehat{q}_{k}\right)
\end{array}\right.
$$

where $\widehat{q}_{k}$ is the internal state. Following the same reasoning, since $g_{\theta}$ corresponds to the state transition function of an automaton with finite input memory, it is clear that after a transient time of maximal length equal to $M$, it holds that, for $k \geq M$,

$$
\widehat{q}_{k}=q_{k} \text { and } \widehat{z}_{k+b}=z_{k+b} .
$$

In other words, the generators synchronize automatically after at most $M$ iterations. Hence, the decryption is automatically and properly achieved after at most $M$ iterations too. No specific synchronizing protocol between the cipher and the decipher is needed. This explains the terminology Self-Synchronizing Stream Ciphers. The quantity $M$ is called the synchronization delay. 


\subsection{Motivation on the use of LPV systems}

To obtain a finite input memory feature, the solutions proposed in the open literature call for state transition functions $g_{\theta}$ in the form of shifts or more generally $T$-functions ( $T$ for Triangle), which are functions that propagate dependencies in one direction only. Till now, none of the proposed SSSC have involved non triangular state transition functions although $T$-functions are known to suffer from weakness [22]. Indeed, $T$-functions get linear properties which make them easier to cryptanalyse. Furthermore, for the existing $\operatorname{SSSC}([16,9,10])$, the design relied more on ad-hoc approaches than on systematic rules of construction. It is explained by the fact that no systematic methodology for constructing automata with finite input memory and involving general non triangular state transition functions was proposed so far. As a clue to tackle those issues, we first restrict our investigation on the class of linear systems. Let us consider the finite state automaton given by

$$
\begin{aligned}
\left\{\begin{aligned}
q_{k+1} & =P q_{k}+Q c_{k+b}, \\
z_{k+b} & =R q_{k}
\end{aligned}\right. \\
c_{k+b}=z_{k+b}+S m_{k}
\end{aligned}
$$

and

$$
\begin{aligned}
& \left\{\begin{array}{l}
\widehat{q}_{k+1}=P \widehat{q}_{k}+Q c_{k+b}, \\
\widehat{z}_{k+b}=R \widehat{q}_{k}
\end{array}\right. \\
& \widehat{m}_{k+b}=S^{-1}\left(c_{k+b}-\widehat{z}_{k+b}\right) .
\end{aligned}
$$

where $P, Q, R$ and $S$ are square matrices. Their entries belong to the set $A$. Assume that the following condition holds:

$$
\exists K \in \mathbb{N} \text {, such that } P^{l}=0 \text { for any } l \geq K .
$$

In other words, if the matrix $P$ is nilpotent (the integer $K$ is the index of nilpotency of $P$ ), then after a finite number $K$ of iterations of (9) (resp. (11)), $q_{k}$ (resp. $\left.\widehat{q}_{k}\right)$ becomes independent of the initial condition $q_{0}\left(\right.$ resp. $\left.\hat{q}_{0}\right)$ and so, the automaton (9) (resp. (11)) has finite input memory, as required for an SSSC. Equations (9) and (10) (resp. (11) and (12)) define respectively the cipher and the decipher part of an SSSC. The following correspondences apply:

- $z_{k+b}=R q_{k}$ plays the role of the keystream symbol of the cipher;

- $\widehat{z}_{k+b}=R \widehat{q}_{k}$ plays the role of the keystream symbol of the decipher;

- the function $\left(z_{k+b}, m_{k}\right) \mapsto z_{k+b}+S m_{k}$ plays the role of $e$ (encryption function);

- the function $\left(\widehat{z}_{k+b}, c_{k+b}\right) \mapsto S^{-1}\left(c_{k+b}-\widehat{z}_{k+b}\right)$ plays the role of $d$ (decryption function);

- the integer $K$ plays the role of the synchronization delay $M$. 
Thereby, the design of a linear SSSC would be straightforward by choosing a nilpotent matrix $P$ and arbitrary matrices $Q, R$ and $S$. Unfortunately, linear dynamics are prohibited in cryptography because in such a case, it is well admitted that cryptanalysis is easy. To simply obtain nonlinear systems (with potentially complex nonlinearities as seen later), we propose here a structure inspired from the linear one. We replace a prescribed number of timeinvariant entries of the matrices of Equations (9-10) and (11-12) (in particular matrix $P$ ) by time-varying parameters. If those parameters denoted by $\rho^{i}(k)\left(i=1, \ldots, L_{\rho}\right.$ with $L_{\rho}$ the number of time-varying parameters $)$ get the form $\rho^{i}(k)=\varphi^{i}\left(c_{k}, c_{k-1}, \cdots, c_{k-s}\right)(s \in \mathbb{N})$ where every $\varphi^{i}$ is a nonlinear function, the resulting system becomes nonlinear. It belongs to the class of Linear Parameter-Varying (LPV) systems. Let us note that the functions $\varphi^{i}$ have the same form as the canonical equation of an SSSC (see (6)). It means that such an SSSC admits a recursive architecture. This architecture is new regarding the literature and differs from the serial and parallel ones proposed in the $90 \mathrm{~s}$ by Maurer [19].

The point is that, the matrix raised to the power of $l$, involved in condition (13) and guaranteeing an automaton with finite input memory in the linear case, must be replaced by the ordered product of $l$ time-varying matrices in the LPV case. Following the linear case, a direct design would consist in choosing parameter-dependent matrices such that the ordered product of $l$ matrices is zero for $l \geq K$, for any initial condition and any values of $\rho^{i}(k)$. A non triangular state transition function would be simply obtained by non triangular matrices which do not share a common triangular basis. However, that raises a new problem. Indeed, it is closely related to the problem of mortality. It is said that a set of matrices is mortal if the zero matrix can be expressed as the product of a finite number of matrices. And yet, from the papers [23, 4, 6], except from some very special cases, the problem of checking mortality of a set of matrices is undecidable. The problem under consideration here is even more challenging since the matrices are not given but must be chosen. In other words, we are not concerned with analysis but with synthesis.

Summarizing our purpose, we are seeking for a systematic methodology to build a family of SSSC. This can be done by designing LPV automata in the form (9-10) and (11-12) where the linear matrices are substituted by parameter dependent matrices involving nonlinear functions $\varphi^{i}$ of the shifted cryptograms $c_{k}$. A direct design cannot be achieved because it is related to the intricate problem of mortality. In the sequel, it is shown how to tackle this problem. The key idea is, first, to build a flat LPV system. Next, from this flat LPV system, an automaton with finite input memory can be systematically obtained by calculating its left inverse. It is recalled that in control theory, given a dynamical system and considering an input sequence and its corresponding output response, its left inverse is a system that reproduces the input sequence when it is forced by the output response. This connection between flatness and SSSC may appear as quite natural, but is new, regarding the literature. It is detailed in next section. 


\section{Connection between flat systems and SSSC}

Difference flatness is the discrete-time counterpart of differential flatness, a property of some continuous-time controlled dynamical systems introduced in [15]. For a flat discrete-time system, the state variables as well as the input are written as a function of the flat output (including forward and backward shifts in the output). The reader can refer to the book [25] for an introduction on difference flatness and some applications. However, it deals with linear systems. Results on difference flatness for LPV systems have never been published before the paper [13] which is the source of the present paper (Subsections 3.1 and 3.2 are recalled from [13]). Since in this paper, only discrete-time dynamical systems are considered, the word flatness will be often used for short but without confusion.

\subsection{Definition of difference flatness for LPV systems}

Let us consider a Single Input Single Output (SISO) Linear ParameterVarying (LPV) system denoted by $\Sigma_{\rho}$, defined over a field $\mathbb{F}$, described by the following state space representation:

$$
\Sigma_{\rho}:\left\{\begin{aligned}
x_{k+1} & =A_{\rho(k)} x_{k}+B_{\rho(k)} m_{k} \\
c_{k} & =C_{\rho(k)} x_{k}+D_{\rho(k)} m_{k}
\end{aligned}\right.
$$

where $k \in \mathbb{N}$ stands for the discrete time, $x_{k} \in \mathbb{F}^{n}$ is the state vector, $m_{k} \in \mathbb{F}$ is the input, $c_{k} \in \mathbb{F}$ is the output. The matrices $A \in \mathbb{F}^{n \times n}, B \in \mathbb{F}^{n \times 1}, C \in \mathbb{F}^{1 \times n}$ and $D \in \mathbb{F}^{1 \times 1}$ are respectively the dynamical matrix, the input matrix, the output matrix and the direct transfer matrix. Such a system is called Linear Parameter-Varying because it is written with a linear dependency with respect to the state vector. The set of all varying parameters of $A, B, C$ and $D$ are collected on a vector denoted by $\rho(k)=\left[\rho^{1}(k), \rho^{2}(k), \ldots, \rho^{L_{\rho}}(k)\right] \in \mathbb{F}^{L_{\rho}}$ where $L_{\rho}$ is the total number of non-zero (possibly varying) entries. The matrices $A$, $B, C$ and $D$ do not necessarily depend on all the parameters $\rho^{i}(k)$. Such systems can exhibit nonlinear dynamics. Indeed, the nonlinearity is obtained by defining the varying parameters $\rho^{i}(k)$ as nonlinear functions $\varphi^{i}: \mathbb{F}^{s+1} \rightarrow \mathbb{F}$ of the output $c_{k}$ (or a finite number of shifts) $\rho^{i}(k)=\varphi^{i}\left(c_{k}, c_{k-1}, \cdots, c_{k-s}\right.$ ) with $s$ a natural number. Let us note that the notation $\rho^{i}(k)$ (usual in the literature for LPV systems) is somehow abusive because it does not reflect an explicit dependency with respect to the time $k$ but on quantities, here $c_{k}$, indexed with $k$. Usually, in the context of cryptography, the systems operate on the $q$ elements finite field $\mathbb{F}_{q}$ where $q$ is a prime power. Hence, in this context, LPV systems can be considered as hybrid systems, and more precisely, switched linear systems with $q^{L_{\rho}}$ modes since every $\rho^{i}(k)$ takes values in a finite set.

Now, let us go into more detail. For a non negative integer $k$, a sequence $\{\rho(k), \rho(k+1), \ldots\}$ will be called a realization of varying parameters and denoted with $\rho$. 
Definition 1. The system (14) is flat, if for every realization $\rho$, there exists a variable $c_{k}$, called flat output, such that all system variables can be expressed as a function of the flat output and a finite number of its backward and forward shifts. In other words, there exist two functions $\mathcal{F}_{\rho}$ and $\mathcal{G}_{\rho}$ parametrized by $\rho$ such that

$$
\left\{\begin{aligned}
x_{k} & =\mathcal{F}_{\rho}\left(c_{k+k_{\mathcal{F}}}, \ldots, c_{k+k_{\mathcal{F}}^{\prime}}\right) \\
m_{k} & =\mathcal{G}_{\rho}\left(c_{k+k_{\mathcal{G}}}, \ldots, c_{k+k_{\mathcal{G}}^{\prime}}\right)
\end{aligned}\right.
$$

where $k_{\mathcal{F}}, k_{\mathcal{F}}^{\prime}, k_{\mathcal{G}}$ and $k_{\mathcal{G}}^{\prime}$ are $\mathbb{Z}$-valued integers.

\subsection{Conditions for flat outputs}

First, it must be pointed out that for a given dynamics (first equation of (14)), whether $c_{k}$ is a flat output or not necessarily depends on the matrices $C$ and $D$. Besides, for some specific matrices $A$ and $B$, it may happen that none of the matrices $C$ and $D$ yield to a flat output. As a result, the conditions which guarantee that an output of (14) is flat must be expressed in terms of properties verified by the 4 -tuple of state matrices $(A, B, C, D)$. Before proceeding further, it is necessary to deal with the relative degree of (14). We recall below a usual general definition.

Definition 2. The relative degree of a SISO discrete-time dynamical system is the minimal number $r$ of iterations such that the output $c_{k+r}$ at time $k+r$ is sensitive to the input $m_{k}$.

Now, let us restrict this general definition to the system defined by equation (14). To this end, for $k_{2} \geq k_{1}$, let us denote by $\prod_{l=k_{2}}^{k_{1}} A_{\rho(l)}$ the product

of matrices $A_{\rho(l)}$ from $k_{2}$ to $k_{1}$. For $k_{2}<k_{1}, \prod_{l=k_{2}}^{k_{1}} A_{\rho(l)}=\mathbf{1}_{n}$ (the identity matrix of dimension $n)$. Let us introduce the quantity $\mathcal{T}_{\rho(k)}^{i, j}$ defined for $j \leq i$ as

$$
\begin{aligned}
& \mathcal{T}_{\rho(k)}^{i, j}=C_{\rho(k+i)} \prod_{l=k+i-1}^{k+j+1} A_{\rho(l)} B_{\rho(k+j)} \text { if } j \leq i-1 \text { and } \\
& \mathcal{T}_{\rho(k)}^{i, i}=D_{\rho(k+i)} .
\end{aligned}
$$

From (14), it holds that $c_{k+1}=C_{\rho(k+1)} A_{\rho(k)} x_{k}+\mathcal{T}_{\rho(k)}^{1,0} m_{k}+\mathcal{T}_{\rho(k+1)}^{0,0} m_{k+1}$. Then, by iterating the output $c_{k}$ and noting that $\mathcal{T}_{\rho(k+1)}^{i, j}=\mathcal{T}_{\rho(k)}^{i+1, j+1}$ for $i \geq 0$, it follows that

$$
c_{k+i}=C_{\rho(k+i)} \prod_{l=k+i-1}^{k} A_{\rho(l)} x_{k}+\sum_{j=0}^{i} \mathcal{T}_{\rho(k)}^{i, j} m_{k+j} .
$$

Hence, if (14) admits a finite relative degree $r$, it follows from Definition 2 that it is:

1. $r=0$ if $\mathcal{T}_{\rho(k)}^{0,0} \neq 0$ for any realization $\rho$;

2. the smallest non-zero integer $r$ such that for any realization $\rho$;

$$
\begin{gathered}
\mathcal{T}_{\rho, j}^{i, j}=0 \\
\mathcal{T}_{\rho(k)}^{r, 0} \neq 0 .
\end{gathered} \quad \text { for } i=0, \ldots, r-1 \text { and } j=0, \ldots, i,
$$


Hence, it holds that

$$
c_{k+r}=C_{\rho(k+r)} \prod_{l=k+r-1}^{k} A_{\rho(l)} x_{k}+\mathcal{T}_{\rho(k)}^{r, 0} m_{k} .
$$

In the sequel, to make the notation less cluttered, we will merely write $\mathcal{T}$ instead of $\mathcal{T}_{\rho(k)}^{r, 0}$.

Proposition 1. If the LPV system (14) has a relative degree $r<\infty$, the following condition involving product of matrices, fulfilled for a positive integer $K$ and any realization $\rho$

$$
P_{\rho(k+K-1: k+K-1+r)} P_{\rho(k+K-2: k+K-2+r)} \cdots P_{\rho(k: k+r)}=0
$$

with

$$
P_{\rho(k: k+r)}=A_{\rho(k)}-B_{\rho(k)} \mathcal{T}^{-1} C_{\rho(k+r)} \prod_{l=k+r-1}^{k} A_{\rho(l)}
$$

is equivalent to $c_{k}$ is a flat output.

Proof 1. If the LPV system (14) has a relative degree $r<\infty$, the following dynamical system can always be defined since $\mathcal{T}$ is non-zero.

$$
\left\{\begin{array}{l}
q_{k+1}=P_{\rho(k: k+r)} q_{k}+B_{\rho(k)} \mathcal{T}^{-1} c_{k+r} \\
\widehat{m}_{k+r}=\mathcal{T}^{-1}\left(C_{\rho(k+r)} \prod_{l=k+r-1}^{k} A_{\rho(l)} q_{k}-c_{k+r}\right)
\end{array}\right.
$$

Iterating $K-1$ times the first equation of (22) yields

$$
\begin{aligned}
& x_{k}=P_{\rho(k-1: k-1+r)} \cdots P_{\rho(k-K: k-K+r)} x_{k-K}+ \\
& \sum_{i=1}^{K-1}\left[\prod_{j=0}^{i-1} P_{\rho(k-j-1: k+r-j-1)}\right] \mathcal{T}^{-1} B_{\rho(k-i-1)} c_{k-i-1+r} .
\end{aligned}
$$

Let us define $\varepsilon_{k}=x_{k}-q_{k}$. By simple manipulations, it can be shown that $\varepsilon_{k}$ verifies $\varepsilon_{k+1}=P_{\rho(k: k+r)} \varepsilon_{k}$. Hence, after a finite transient time equal to $K$, since (20) holds, one has $q_{k}=x_{k}$. Hence, since the parameters $\rho^{i}(k)$ depend on shifted outputs, the state vector $x_{k}$ exclusively depends on shifted outputs if and only if (20) holds. Finally, $x_{k}$ reads

$$
x_{k}=\sum_{i=0}^{K-1}\left[\prod_{j=0}^{i-1} P_{\rho(k-j-1: k+r-j-1)}\right] \mathcal{T}^{-1} B_{\rho(k-i-1)} c_{k-i-1+r},
$$

which gives the explicit function $\mathcal{F}_{\rho}$ involved in (15). Letting $\omega_{k}=m_{k}-\widehat{m}_{k+r}$ and following the same reasoning, it is shown that $m_{k}$ exclusively depends on shifted outputs. The explicit form of $\mathcal{G}_{\rho}$ involved in (15) is obtained by substituting (24) into the second equality of (22). It shows that the system defined by Equation (14) is flat with $c_{k}$ as flat output if and only if (20) holds.

Remark 1. The system defined by Equation (22) is nothing but the left inverse system of the LPV system (14). 


\subsection{Connection}

In this subsection, we bring out the connection between flat LPV systems and SSSC.

Proposition 2. If the LPV system (14) has relative degree $r<\infty$ and is flat, then the finite state automata given by

$$
\begin{aligned}
\left\{\begin{aligned}
q_{k+1}= & P_{\rho(k: k+r)} q_{k}+B_{\rho(k)} \mathcal{T}^{-1} c_{k+r}, \\
z_{k+r}= & C_{\rho(k+r)} \prod_{l=k+r-1}^{k} A_{\rho(l)} q_{k}
\end{aligned}\right. \\
c_{k+r}=z_{k+r}+\mathcal{T} m_{k}
\end{aligned}
$$

and

$$
\left\{\begin{array}{c}
\widehat{q}_{k+1}=P_{\rho(k: k+r)} \widehat{q}_{k}+B_{\rho(k)} \mathcal{T}^{-1} c_{k+r}, \\
\widehat{z}_{k+r}=C_{\rho(k+r)} \prod_{l=k+r-1}^{k} A_{\rho(l)} \widehat{q}_{k} \\
\widehat{m}_{k+r}=\mathcal{T}^{-1}\left(c_{k+r}-\widehat{z}_{k+r}\right)
\end{array}\right.
$$

define an SSSC.

Proof 2. If the system (14) has relative degree $r<\infty$ and is flat, then $\mathcal{T}$ is non-zero and thus, systems (25-26) and (27-28) are well defined. Systems (25) and (27) are the left inverse system of (14) as emphasized in Remark 1. The property (20), that is flatness, ensures that systems (25) and (27) are automata with finite input memory. They can respectively be identified with the system (3) and with the system (7). Identifying (26) with (1) and (28) with (2) gives respectively the encryption and the decryption function.

The following correspondences hold:

- $z_{k+r}=C_{\rho(k+r)} \prod_{l=k+r-1}^{k} A_{\rho(l)} q_{k}$ plays the role of the keystream symbol of the cipher;

- $\widehat{z}_{k+r}=C_{\rho(k+r)} \prod_{l=k+r-1}^{k} A_{\rho(l)} \widehat{q}_{k}$ plays the role of the keystream symbol of the decipher;

- $r$ (relative degree) plays the role of $b$ (delay);

- the function $\left(z_{k+r}, m_{k}\right) \mapsto z_{k+r}+\mathcal{T} m_{k}$ plays the role of $e$ (encryption function);

- the function $\left(\widehat{z}_{k+r}, c_{k+r}\right) \mapsto \mathcal{T}^{-1}\left(c_{k+r}-\widehat{z}_{k+r}\right)$ plays the role of $d$ (decryption function);

- the integer $K$ plays the role of the synchronization delay $M$.

It is recalled that the nonlinearity is obtained by defining the values of every varying parameters $\rho^{i}(k)\left(i=1, \ldots, L_{\rho}\right)$ involved in the matrices of $(25$ 28) as nonlinear functions $\varphi^{i}$ of a finite number of past cryptograms $\left(\rho^{i}(k)=\right.$ $\left.\varphi^{i}\left(c_{k}, c_{k-1}, \cdots, c_{k-s}\right)\right)$. In cryptography, those functions are called S-boxes. 
Remark 2. The encryption function e and the decryption functions d are quite simple. This is a common feature for SSSC. For example in the Boolean case, those functions are often nothing but the exclusive or. Actually, the security is essentially based on the properties of the keystream sequences delivered by (25) and (27).

To sum up, as an alternative to a direct design of automata with finite input memory required for SSSC, which is in general impossible because related to the problem of mortality, an indirect approach has been proposed. It consists, first, on the design of a flat LPV system (14). Next, we deduce the automata (25) and (27) by calculating the left inverse system of the LPV system. It is central to emphasize that since the LPV system is flat, the automata are necessarily with finite input memory. The outcome of such an approach is that the matrix $P_{\rho(k: k+r)}$ involved in (25) and (27) define a state transition function which is not necessarily triangular and thus, may represent a larger class than $T$-functions. This approach is new in cryptography.

In our context, the flatness property must be guaranteed for any non-zero entries of the matrices involved in (25) and (27). Indeed, some entries correspond to time-varying coefficients, the other ones are constant entries. But all of these entries are parametrized by the secret key $\theta$ which ranges in the key space $\mathcal{K}$, a huge set of values. All in all, flatness must be ensured from a structural point of view. Hence, structural analysis can be a natural and relevant solution to construct structural flat systems. The construction is detailed in next section.

\section{Construction of structural flat systems and SSSC}

\subsection{Structured systems and digraphs}

The core idea is that the LPV system (14) can be considered as an admissible realization of a corresponding structured linear system. A structured linear system is a system only defined by the sparsity pattern of the state space realization matrices. In other words, for a structured linear system, we distinguish between the entries that are fixed to zero and the other ones that can take any value in $\mathbb{F}$, including the ones which are time-varying. Hence, a structured linear discrete-time system is a system that admits the form:

$$
\Sigma_{\Lambda}: x_{k+1}=I_{A} x_{k}+I_{B} m_{k}
$$

The entries of the matrices of $\Sigma_{\Lambda}$ are '0' or ' 1 '. In particular, the entries $A(i, j)$ of $I_{A}$ (resp. $B(i)$ of $I_{B}$ ) that are '0' means that there are no relation (dynamical interaction) between the state $x_{k+1}^{i}$ at time $k+1$ and the state $x_{k}^{j}$ at time $k$ (resp. the state $x_{k+1}^{i}$ at time $k+1$ and the input $m_{k}$ at time $k$ ). The entries that are '1' means that there is a relation. As a simple example, let us consider an LPV system with the setting

$$
A_{\rho(k)}=\left(\begin{array}{cc}
a & 0 \\
1 & \rho^{1}(k)
\end{array}\right) \text { and } B_{\rho(k)}=\left(\begin{array}{c}
\rho^{2}(k) \\
0
\end{array}\right)
$$


where $a$ is a constant element in $\mathbb{F}, \rho^{1}(k)$ and $\rho^{2}(k)$ are varying parameters in $\mathbb{F}$. The dynamical matrix and the input matrix $I_{A}$ and $I_{B}$ of the corresponding structured linear system read:

$$
I_{A}=\left(\begin{array}{ll}
1 & 0 \\
1 & 1
\end{array}\right), I_{B}=\left(\begin{array}{l}
1 \\
0
\end{array}\right)
$$

As a consequence, if the structural linear system (29) derived from (14) is flat, the flatness will hold for any realization $\rho$ of (14). Hence, the challenge is to define a methodology to construct flat linear structural systems. A graphbased approach is suggested below to that purpose. It is based on the result provided in [21]. Let us recall some basic requirements on digraphs. A digraph $\mathcal{G}\left(\Sigma_{\Lambda}\right)$ describing the structured linear system associated to the state equations $(14)$, is the combination of a vertex set $\mathcal{V}$ and an edge set $\mathcal{E}$. The vertices represent the states and the input components of $\Sigma_{\Lambda}$ while the edges describe the dynamic relations between these variables. One has $\mathcal{V}=\mathbf{X} \cup\{\mathbf{m}\}$ where $\mathbf{X}$ is the set of state vertices defined as $\mathbf{X}=\left\{\mathbf{x}^{\mathbf{1}}, \ldots, \mathbf{x}^{\mathbf{n}}\right\}$ and $\mathbf{m}$ is the input vertex. The edge set is $\mathcal{E}=\mathcal{E}_{A} \cup \mathcal{E}_{B}$, with $\mathcal{E}_{A}=\left\{\left(\mathbf{x}^{\mathbf{i}}, \mathbf{x}^{\mathbf{j}}\right) \mid A(i, j) \neq 0\right\}$ and $\mathcal{E}_{B}=\left\{\left(\mathbf{m}, \mathbf{x}^{\mathbf{i}}\right) \mid B(i) \neq 0\right\}$. They also correspond to the weights of the edges of the digraph. In the sequel, we will denote by $\mathbf{v}^{\mathbf{j}},(j=0, \ldots, n)$ a vertex of the digraph $\mathcal{G}\left(\Sigma_{\Lambda}\right)$ regardless whether it is the input or a state vertex.

- A directed path $\mathbf{P}$ is a sequence of successive edges directed in the same direction which connect a sequence of vertices. It is said that the path $\mathbf{P}$ covers a vertex if this vertex is the begin or the end vertex of one of the edges of $\mathbf{P}$;

- In a directed path from a vertex $\mathbf{v}^{\mathbf{i}}$ to a vertex $\mathbf{v}^{\mathbf{j}}$, it is said that $\mathbf{v}^{\mathbf{j}}$ is a successor of $\mathbf{v}^{\mathbf{i}}$ and conversely, $\mathbf{v}^{\mathbf{i}}$ is a predecessor of $\mathbf{v}^{\mathbf{j}}$;

- A simple path is a path which contains no repeated vertices;

- The length of a directed path $\mathbf{P}$ is equal to the number of edges involved in $\mathbf{P}$. We let $\ell\left(\mathbf{v}^{\mathbf{i}}, \mathbf{v}^{\mathbf{j}}\right)$ denote the minimal length of a path connecting $\mathbf{v}^{\mathbf{i}}$ to $\mathbf{v}^{\mathbf{j}}$

- $V_{\text {ess }}\left(\mathbf{v}^{\mathbf{i}}, \mathbf{v}^{\mathbf{j}}\right)$ is the set of vertices, called essential vertices from $\mathbf{v}^{\mathbf{i}}$ to $\mathbf{v}^{\mathbf{j}}$, which are common to all the paths connecting $\mathbf{v}^{\mathbf{i}}$ to $\mathbf{v}^{\mathbf{j}}$.

Remark 2. In graph theory, the terminology may be different according to authors. A directed path as defined here may be called a walk. A simple path as defined here may be merely called a path.

We recall from [21] the necessary and sufficient conditions which must be satisfied for any vertex $\mathbf{v}^{\mathbf{i}}(i \in\{1, \ldots, n\})$ of the digraph $\mathcal{G}\left(\Sigma_{\Lambda}\right)$ to be associated to a flat output $c_{k}^{F}=x_{k}^{i}(i \in\{1, \ldots, n\})$ of (14). In such a case, the output state space matrix $C_{\rho(k)}=C$ is constant and has zero entries except the entry located at the column number $i$ which is equal to one. The direct transfer matrix $D_{\rho(k)}=D$ is the zero matrix. 
Theorem 1. The output $c_{k}^{F}=x_{k}^{i}(i \in\{1, \ldots, n\})$ of the structured linear system (29), associated to the vertex $\mathbf{v}^{\mathbf{F}} \in \mathbf{X}$ in the associated digraph $\mathcal{G}\left(\Sigma_{\Lambda}\right)$, is generically a flat output iff, the three following conditions hold:

CO. $\mathbf{v}^{\mathbf{F}}$ is a successor of $\mathbf{m}$;

C1. Any simple paths from $\mathbf{m}$ to $\mathbf{v}^{\mathbf{F}}$ have the same length equal to $\ell\left(\mathbf{m}, \mathbf{v}^{\mathbf{F}}\right)$;

C2. Any cycles cover at least an element of $V_{\text {ess }}\left(\mathbf{m}, \mathbf{v}^{\mathbf{F}}\right)$.

Actually, as shown in [21], Conditions C0-C2 ensure that the state and the input of the structured linear system (29) do no longer depend on the initial state after a finite transient time regardless of the weights of the edges. Consequently, it is shown that they can be exclusively expressed as some functions of shifted outputs parametrized by the weights of the edges. Since a weight is a constant or a function $\varphi^{i}$ of shifted outputs (in our context, the shifted cryptograms), both the state and the input depend exclusively on shifted outputs (here, the cryptograms). Hence, Conditions C0-C2 are instrumental for the construction of structural flat dynamical systems. A systematic construction of digraphs fulfilling $\mathbf{C 0}-\mathbf{C 2}$ is proposed below.

\subsection{Construction of flat LPV systems}

The digraph $\mathcal{G}\left(\Sigma_{\Lambda}\right)$ related to the system $\Sigma_{\Lambda}$ of dimension $n$ involves $n+1$ vertices. The input is assigned to the vertex denoted by $\mathbf{v}^{\mathbf{0}}$. The other $n$ vertices are denoted by $\mathbf{v}^{\mathbf{1}}, \ldots, \mathbf{v}^{\mathbf{n}}$. Let $\mathbf{v}^{\mathbf{r}}$ be the vertex that corresponds to the flat output $\mathbf{v}^{\mathbf{r}}$, that is $\mathbf{v}^{\mathbf{F}}=\mathbf{v}^{\mathbf{r}}$.

Step 1: For, $i=0, \ldots, n-1$, add the edges $\left(\mathbf{v}^{\mathbf{i}}, \mathbf{v}^{\mathbf{i}+\mathbf{1}}\right)$. There are $r$ edges which connect $\mathbf{v}^{\mathbf{0}}$ to $\mathbf{v}^{\mathbf{r}}$. Hence, the relative degree of the corresponding structured dynamical system is $r$.

After Step 1, Conditions C0-C2 are fulfilled for $\mathbf{v}^{\mathbf{F}}=\mathbf{v}^{\mathbf{r}}$. However, this line topology corresponds to quite trivial dynamical systems since it corresponds to state transition functions in the form of simple shifts. Let us recall that we aim at designing an automaton possibly involving state transition functions more general than $T$-functions. A shift is a special and trivial $T$-function. To this end, the following steps provide a way of adding edges $\left(\mathbf{v}^{\mathbf{i}}, \mathbf{v}^{\mathbf{j}}\right)$ while guaranteeing that Conditions C0-C2 are still fulfilled.

Step 2: Add the edges $\left(\mathbf{v}^{\mathbf{r}+\mathbf{i}}, \mathbf{v}^{\mathbf{r}+\mathbf{i}+\mathbf{1}}\right)$ for $i=1, \ldots, n-r-1$. Step 2 allows vertex $\mathbf{v}^{\mathbf{j}}, j=r+1, \ldots, n$ to have a predecessor. Indeed, if not so, the dynamics of the corresponding vertex $\mathbf{v}^{\mathbf{j}}$ would reduce to $x_{k+1}^{j}=0$ and would be clearly useless. The resulting path will be called main directed path and is depicted in Figure 1.

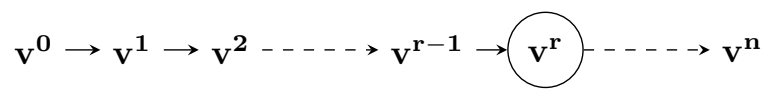

Figure 1: Digraph obtained after completion of Step 1-2. The vertex $\mathbf{v}^{\mathbf{r}}$ corresponds to the flat output 
Step 3: Add the edges $\left(\mathbf{v}^{\mathbf{r}}, \mathbf{v}^{\mathbf{i}}\right), i=1, \ldots, n$ that connect the vertex $\mathbf{v}^{\mathbf{r}}$ to any other vertices of the graph (except the vertex $\mathbf{v}^{\mathbf{0}}$ related to the input).

Step 4: For every vertex $\mathbf{v}^{\mathbf{i}}, i=1, \ldots, r-1$, add the directed edge $\left(\mathbf{v}^{\mathbf{i}}, \mathbf{v}^{\mathbf{j}}\right)$ for $j=1, \ldots, i$. Note that those edges introduce cycles, including cycles of order 1 , but those cycles satisfy Condition $\mathbf{C 2}$ since $\mathbf{v}^{\mathbf{i}}$, for $i=0, \ldots, r-1$, belong to $V_{\text {ess }}\left(\mathbf{m}, \mathbf{v}^{\mathbf{r}}\right)$.

The graph obtained after Step 1-4 is depicted in Figure 2.

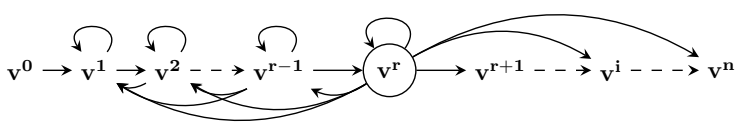

Figure 2: Graph obtained after Step 1-4.

Step 5: For every vertex $\mathbf{v}_{\mathbf{i}}, i=r+1, \ldots, n$, add the directed edge $\left(\mathbf{v}_{\mathbf{i}}, \mathbf{v}_{\mathbf{j}}\right)$ for $j=1, \ldots, r$ and $j=i+2, \ldots, n$. Let us note that this step generates again cycles but Condition $\mathbf{C 2}$ is still satisfied.

The resulting digraph after completion of Step 1-5 is depicted in Figure 3.

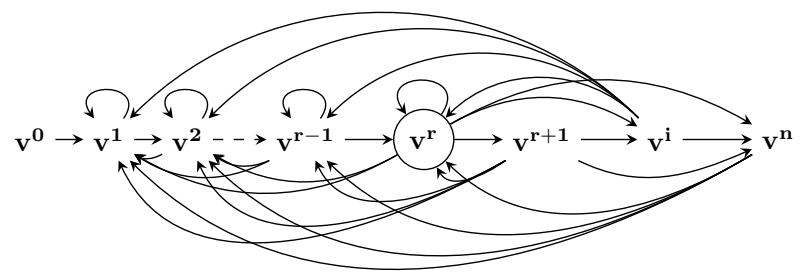

Figure 3: Graph obtained after completion of Step 1-5.

As a result, a digraph is parametrized by the triplet $\left(n, r, n_{a}\right)$. The number of vertices of the digraph is equal to $n+1$ (being $n$ the dimension of the system (29) and (14)). Indeed, there are $n$ vertices assigned to the state components and one assigned to the input. The relative degree $r$ fulfilling (18) gives the number of edges in the main directed path. The integer $n_{a}$ defines the desired number of edges in the digraph $\mathcal{G}\left(\Sigma_{\Lambda}\right)$. It must satisfy $n_{a} \leq n_{M}$, where $n_{M}$ is the maximal number of edges resulting from the construction Step 1-5. A simple counting leads to:

$$
n_{M}=\frac{n(n+1)}{2}+r .
$$

During the construction, at each step, we can decide whether we actually add 
the edges or not. That introduces flexibility in the perspective of providing distinct graphs and thus, distinct SSSC as detailed in Subsection 4.3.

The adjacency matrix, denoted by $\mathcal{I}$, associated to the digraph $\mathcal{G}\left(\Sigma_{\Lambda}\right)$ and obtained after completion of Step 1-5, can be defined as follows. It is the $(n+1) \times(n+1)$ matrix

$$
\mathcal{I}=\left(\begin{array}{c:c}
0 & I_{B}^{t} \\
\hdashline 0 & \underline{B}_{-} \\
\vdots & I_{A}^{t} \\
0 &
\end{array}\right)
$$

where $I_{A}^{t}$ and $I_{B}^{t}$ stands respectively for the transpose of the structured matrices $I_{A}$ and $I_{B}$. The entries $\mathcal{I}_{i j}$ are defined as follows for $1 \leq i, j \leq n$

$$
\mathcal{I}_{i j}=\left\{\begin{array}{l}
1 \text { if there exists an edge from } \mathbf{v}^{\mathbf{j}} \text { to } \mathbf{v}^{\mathbf{i}} \\
0 \text { otherwise. }
\end{array}\right.
$$

The adjacency matrix associated to $\mathcal{G}\left(\Sigma_{\Lambda}\right)$, obtained after completion of Step 1-5, is given by

$\left.\begin{array}{ccccccccccc} & \mathbf{v}^{\mathbf{0}} & \mathbf{v}^{\mathbf{1}} & \mathbf{v}^{\mathbf{2}} & \mathbf{v}^{\mathbf{3}} & \ldots & \mathbf{v}^{\mathbf{r}} & \mathbf{v}^{\mathbf{r}+\mathbf{1}} & \ldots & \mathbf{v}^{\mathbf{n}-\mathbf{1}} & \mathbf{v}^{\mathbf{n}} \\ \mathbf{v}^{\mathbf{0}} & 0 & 1 & 0 & 0 & \cdots & 0 & \cdots & 0 & 0 & 0 \\ \mathbf{v}^{\mathbf{1}} & 0 & 1 & 1 & 0 & \cdots & 0 & \cdots & 0 & 0 & 0 \\ \mathbf{v}^{\mathbf{2}} & 0 & 1 & 1 & 1 & \cdots & 0 & \cdots & 0 & 0 & 0 \\ \mathbf{v}^{\mathbf{3}} & 0 & 1 & 1 & 1 & \cdots & 0 & \cdots & 0 & 0 & 0 \\ \vdots & \vdots & \vdots & \vdots & \vdots & \ddots & 0 & 0 & 0 & 0 & 0 \\ \mathbf{v}^{\mathbf{r}} & 0 & 1 & 1 & 1 & \cdots & 1 & 1 & 1 & 1 & 1 \\ \mathbf{v}^{\mathbf{r}+\mathbf{1}} & 0 & 1 & 1 & 1 & \cdots & 1 & 0 & 1 & 1 & 1 \\ \vdots & \vdots & \vdots & \vdots & \vdots & \vdots & \vdots & \vdots & \ddots & \ddots & \vdots \\ \mathbf{v}^{\mathbf{n}-\mathbf{1}} & 0 & 1 & 1 & 1 & \cdots & 1 & 0 & 0 & 0 & 1 \\ \mathbf{v}^{\mathbf{n}} & 0 & 1 & 1 & 1 & \cdots & 1 & 0 & 0 & 0 & 0\end{array}\right)$

This being the case, we could reformulate Step 1-5 in terms of adjacency matrix. However, the design has been given in terms of a digraph construction because it appears as more natural since Conditions C0-C2 involve a digraph consideration.

The computational complexity of the algorithms for checking Condition C0C2 is of the same order than the algorithms used for finding successors and predecessors of vertex subsets or for computing essential vertices in a digraph [5]. Thus, the complexity of the construction is polynomial and is $O\left(n^{3}\right)$. The open source software Sagemath (see http://www.sagemath.org/) is appropriate to 
construct the digraph $\mathcal{G}\left(\Sigma_{\Lambda}\right)$.

We have summed up after Remark 2 the main line of the design of an SSSC and have raised the problem of constructing a structural flat system. Now, being this issue fixed, next subsection aims at giving the steps for a complete design of SSSC leading to Equations (25)-(28). It is illustrated with a simple example for a clear understanding. A realistic example will be given in Section ??.

\subsection{Construction of SSSC}

Step S1: Choose a triplet $\left(n, r, n_{a}\right)$. Since the flat output $c_{k}^{F}$ is one component $x_{k}^{i}(i=1, \ldots, n)$, it follows that $C_{\rho(k)}=\left(\begin{array}{llllll}0 & \cdots & 0 & 1 & 0 & \ldots\end{array}\right)$ (the only entry 1 is located at the $r^{t h}$ column) and $D_{\rho(k)}=(0 \cdots 0)$. It is recalled that $c_{k}^{F}$ will play the role of the ciphertext.

Example: Consider the triplet $\left(n=2, r=2, n_{a}=5\right)$. As the relative degree $r$ is equal to 2 , it means that the component $x_{k}^{2}$ of the state vector of the corresponding LPV system (14) will be the flat output $c_{k}^{F}$. Hence, $C_{\rho(k)}=C=\left[\begin{array}{ll}0 & 1\end{array}\right]$ and $D_{\rho(k)}=D=0$. 5 .

Step S2: Construct the corresponding digraph $\mathcal{G}\left(\Sigma_{\Lambda}\right)$ according to Step 1-

Example: The graph corresponding to the triplet $\left(n=2, r=2, n_{a}=5\right)$ is depicted in Figure 4.

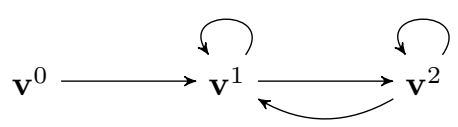

Figure 4: Digraph obtained for $n=2, r=2, n_{a}=n_{M}=5$. The flat output is $c_{k}^{F}=x_{k}^{2}$ and corresponds to the vertex $\mathbf{v}^{2}$

Step S3: Given the digraph $\mathcal{G}\left(\Sigma_{\Lambda}\right)$ characterized by the triplet $\left(n, r, n_{a}\right)$, extract, from the adjacency matrix $\mathcal{I}$, the matrices $I_{A}$ and $I_{B}$ of the structured linear system.

Example: For the digraph $\mathcal{G}\left(\Sigma_{\Lambda}\right)$ depicted in Figure 4, the adjacency matrix $\mathcal{I}$ and the structured matrices $I_{A}$ and $I_{B}$ as defined by (31) are

$$
\mathcal{I}=\left(\begin{array}{lll}
0 & 1 & 0 \\
0 & 1 & 1 \\
0 & 1 & 1
\end{array}\right), I_{A}=\left(\begin{array}{ll}
1 & 1 \\
1 & 1
\end{array}\right), I_{B}=\left(\begin{array}{l}
1 \\
0
\end{array}\right)
$$

Step S4: Replace some of the non-zero entries of $I_{A}$ and $I_{B}$ by a nonlinear function $\rho^{i}(k)=\varphi^{i}\left(c_{k}, c_{k-1}, \cdots, c_{k-s}\right)$ (S-box) to construct the matrices $A_{\rho(k)}$ and $B_{\rho(k)}$ of (14). Not all '1' entries of $I_{A}$ and $I_{B}$ must be assigned to a nonlinear function. Some of them can be merely constant. The choice must obey a tradeoff between complexity of the architecture and security (a matter only discussed 
in a shallow way in Subsection 4.5). Since the construction ensures structural flatness, any choice will preserve the self-synchronization property except for some particular cases which deserve a special treatment. Indeed, it may happen that cryptograms cause $\rho^{i}(k)$ to vanish at time $k$ and thus, the corresponding edge to be cancelled. To preserve a finite and constant relative degree for any $k$, it is sufficient that the weights assigned to the main path connecting the input vertex $\mathbf{v}^{\mathbf{0}}$ to the vertex $\mathbf{v}^{\mathbf{r}}$ are constant.

Example: Let us keep constant and equal to 1 the first three entries of $A$ and let the fourth entry be time-varying. It is denoted by $\rho^{1}(k)$. This finally leads to the following matrices $A_{\rho(k)}$ and $B_{\rho(k)}$ :

$$
A_{\rho(k)}=\left(\begin{array}{cc}
1 & 1 \\
1 & \rho^{1}(k)
\end{array}\right) \text { and } B_{\rho(k)}=B=\left(\begin{array}{l}
1 \\
0
\end{array}\right)
$$

Step S5: Complete the design by deriving the equations of Proposition 2. In particular, calculate the matrix (21) governing the state transition function of the automata (25) and (27).

Example: For the example, one has $P_{\rho(k: k+2)}=A_{\rho(k)}-B \cdot C \cdot A_{\rho(k+1)} \cdot A_{\rho(k)}$. One obtains

$$
P_{\rho(k: k+2)}=\left(\begin{array}{cc}
-\rho^{1}(k+1) & -\rho^{1}(k) \cdot \rho^{1}(k+1) \\
1 & \rho^{1}(k)
\end{array}\right) .
$$

Let us point out that (20) holds for $K=2$. Indeed, it's a simple matter to see that $P_{\rho(k+1: k+3)} P_{\rho(k: k+2)}=0$. It corroborates that $c_{k}$ is a flat output (but it is by construction since the digraph $\mathcal{G}\left(\Sigma_{\Lambda}\right)$ fulfills C0-C2).

The approach proposed here gives thereby a systematic procedure to construct a family of SSSC according to the triplet $\left(n, r, n_{a}\right)$, the type of functions $\varphi^{i}$ and their position in the adjacency matrix. It remains to show that the state transition functions are likely to be non triangular. Next subsection precisely illustrates that, even for this simple example, the state transition matrix $P_{\rho(k: k+2)}$ is non triangular.

\subsection{Non triangular state transition function}

We aim at proving that the matrix $P_{\rho(k: k+2)}$ is not conjugate to a shift or to a $T$-function. To this end, it must be shown that the matrices $P_{\rho(k: k+2)}$, for any realization $\rho$, cannot be simultaneously triangularizable. The characteristic polynomial of $P_{\rho(k: k+2)}$ is given by $N(X)=X\left(X+\left(\rho^{1}(k+1)-\rho^{1}(k)\right)\right)$. Hence, the eigenvalues of $P_{\rho(k: k+2)}$ are 0 and $\rho^{1}(k)-\rho^{1}(k+1)$. It follows that the eigenspace related to the eigenvalues of $P_{\rho(k: k+2)}$ is spanned by:

$$
V_{1}=\left(\begin{array}{c}
-\rho^{1}(k) \\
1
\end{array}\right) \quad \text { and } \quad V_{2}=\left(\begin{array}{c}
-\rho^{1}(k+1) \\
1
\end{array}\right)
$$

And yet, a necessary condition for simultaneous triangularization (see [14]) is that the matrices $P_{\rho(k: k+2)}$ share a common eigenvector for any realization $\rho$. 
As a result, since the parameter $\rho^{1}(k)$ varies, the matrices $P_{\rho(k: k+2)}$ do not fulfill such a requirement. Clearly, even in this trivial example, we have shown that an SSSC with state transition function different from a $T$-function can be obtained.

\subsection{Realistic example and platform}

A realistic algorithm compatible with some central cryptographic considerations has been designed and experimented. Clearly, detailed considerations on security are out of the scope of the paper. However, we give below the main specifications of the SSSC followed by some arguments that motivate the choices.

- Field on which the cryptosystem operates: Galois field $G F(16)$

- Dimension $n$ : 40

- S-boxes $\varphi^{i}$ : the function $c_{k} \longmapsto \frac{1}{c_{k}}+\alpha^{2}$ where $\alpha$ is a primitive element of $G F(16)$

- Relative degree $r: 3$

- Number $n_{a}$ of edges in the digraph $\mathcal{G}\left(\Sigma_{\Lambda}\right): 120$

- Number of S-boxes: 80

The specifications are based on criteria which are, to mention a few, resistance to time-memory trade-off attacks [17], resistance against algebraic attacks [7], good diffusion delay [2] and depth [3].

The open source software Sagemath (see http://www.sagemath.org/) has been used to elaborate the digraph $\mathcal{G}\left(\Sigma_{\Lambda}\right)$ corresponding to the triplet $(n=$ $40, r=3, n_{a}=120$ ). The code is provided in the Appendix (see Algorithm 1). The construction has been performed on an Intel CORE i7 CPU $2.26 \mathrm{GHz}$ running Linux Ubuntu 14.04. All experiments ran single-threaded on the processors. It takes $21 \mathrm{~ms}$ on the computer to obtain the digraph $\mathcal{G}\left(\Sigma_{\Lambda}\right)$.

The platform is a small scale breeding of an ICS-SCADA (Industrial Control Systems - Supervisory Control and Data Acquisition) equipment. It involves several sources of information (temperature sensors, touchscreen, ....). Those information are securely conveyed through a local network, the encryption and decryption being achieved by the way of Arduino MEGA cards involving an ATMEGA 2560 processor. It has a $16 \mathrm{MHz}$ Clock Speed and a $256 \mathrm{~KB}$ Flash Memory. The execution of the encryption and decryption algorithms are performed with a throughput of $8.263 \mathrm{~ms}$. A monitoring system (Figure 7) allows to select the source, the destination and to analyze the self-synchronization performances. 


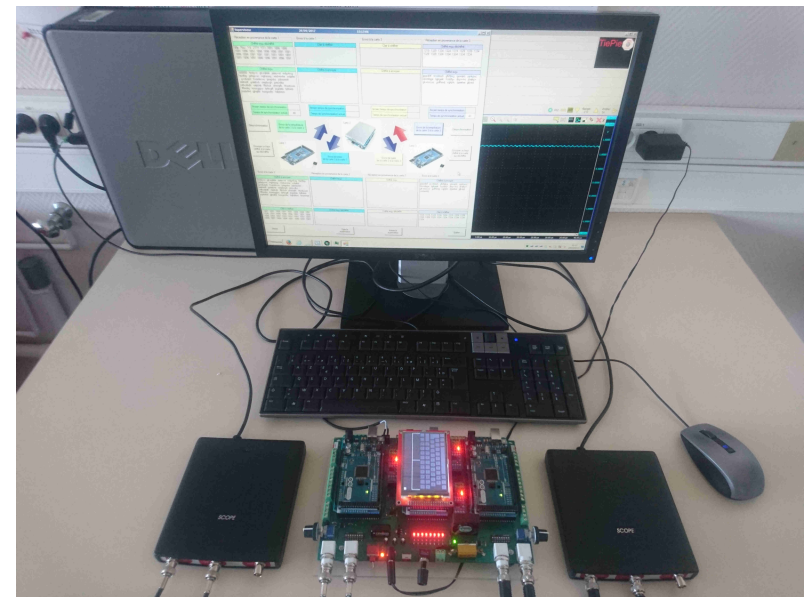

Figure 5: The platform involving the sensors, the touch screen, the Arduino MEGA cards and the oscilloscopes to visualize the analog signals.

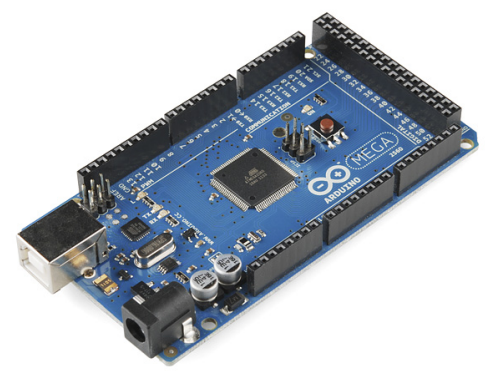

Figure 6: Arduino mega 2560 card

\section{Conclusion}

Cryptography has been investigated as a new field of application of control theory. A systematic and general construction of Self Synchronizing Stream Ciphers based on flat Linear Parameter Varying (LPV) dynamical systems has been proposed. It is based on constructive algebraic and graph-based conditions guaranteeing structural flatness of an LPV system and so self-synchronizing property. It holds for any type of nonlinearities (S-boxes) and values of the secret key. More precisely, a systematic methodology has been proposed in order to obtain SSSC involving non triangular state transition functions. It has been shown that such an approach allows to enlarge the existing classes of SSSC. 


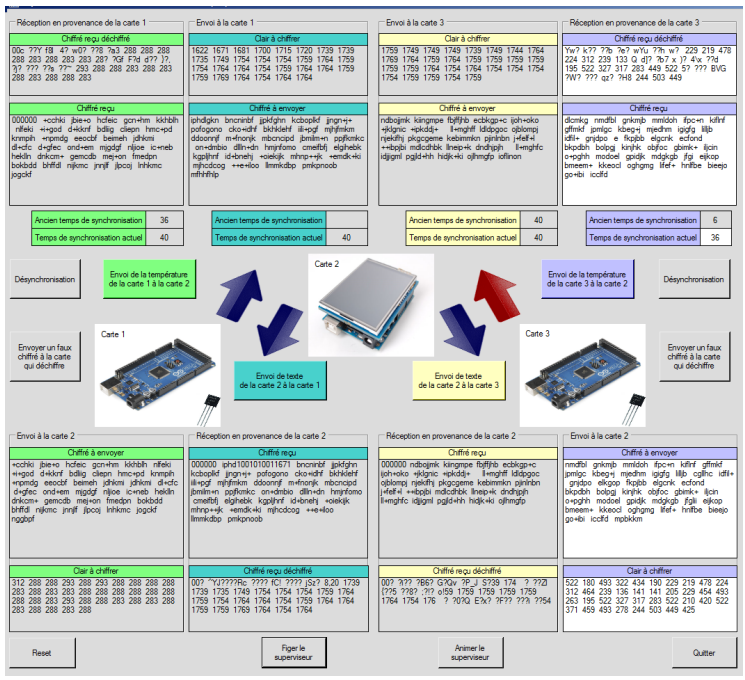

Figure 7: Supervisor

This new structure allows to circumvent the problem raised by the weakness of $T$-functions. Then, a realistic example with detailed specifications has been proposed. As usual in cryptography, we can expect that eavesdroppers will try to break this cryptosystem. Time will tell.

\section{Acknowledgement}

This work was supported by Research Grants ANR-13-INSE-0005-01 from the Agence Nationale de la Recherche in France

\section{Appendix}




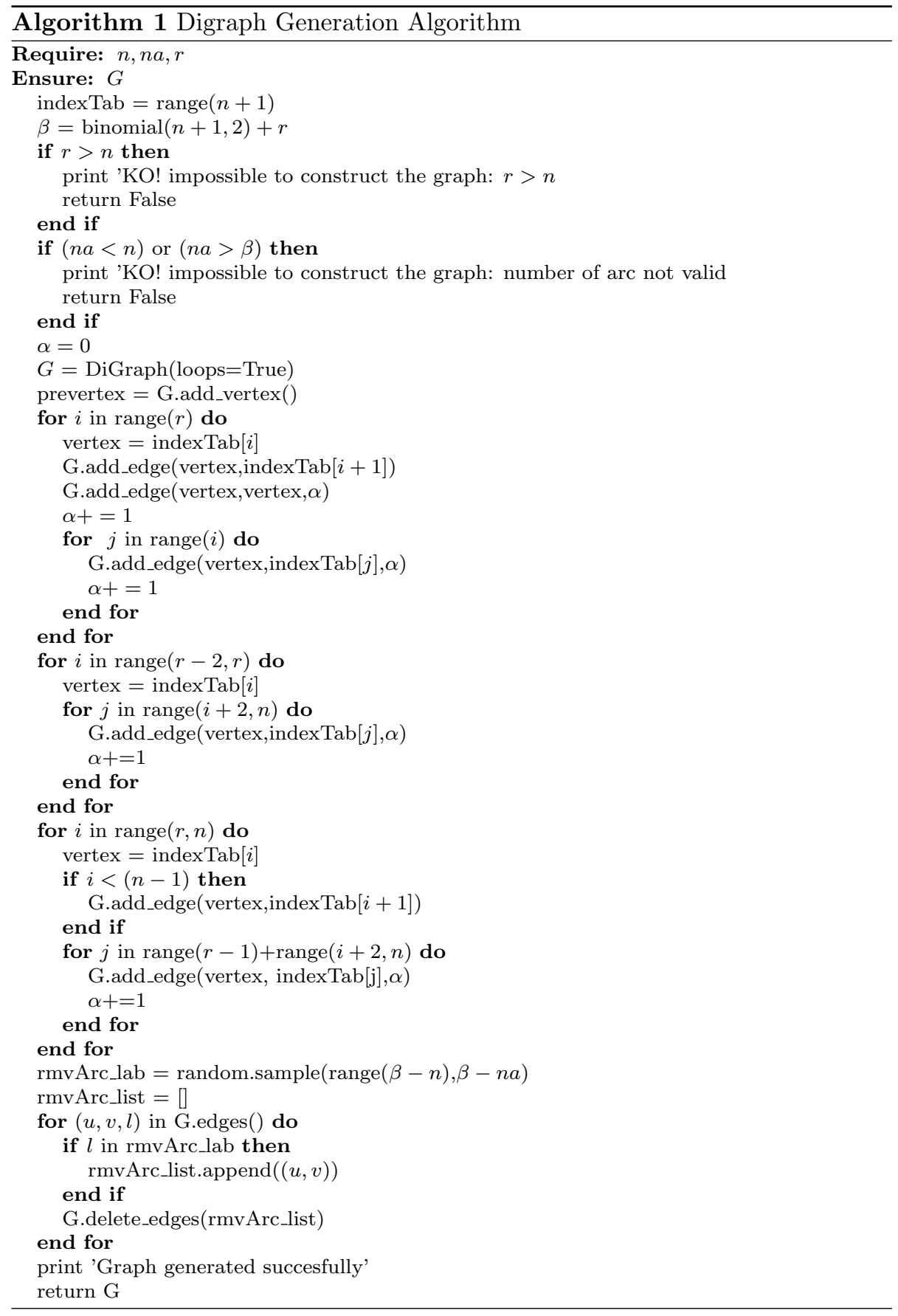

[1] AES, N. (November 26, 2001). FIPS publication 197 - advanced encryption standard. 
[2] Arnault, F., Berger, T. P., Minier, M., and Pousse, B. (2011). Revisiting lfsrs for cryptographic applications. IEEE Transactions on Information Theory, $\mathbf{5 7}(12), 8095-8113$.

[3] Berger, T. P. and Minier, M. (2015). Some results using the matrix methods on impossible, integral and zero-correlation distinguishers for feistel-like ciphers. In A. Biryukov and V. Goyal, editors, Progress in Cryptology - INDOCRYPT 2015 - 16th International Conference on Cryptology in India, Bangalore, India, December 6-9, 2015, Proceedings, volume 9462 of Lecture Notes in Computer Science, pages 180-197. Springer.

[4] Blondel, V. D. and Tsitsiklis, J. N. (1997). When is a pair of matrices mortal? Information Processing Letters, 63, 283-286.

[5] Boukhobza, T. (2010). Partial state and input observability recovering by additional sensor implementation: a graph-theoretic approach. International Journal of Systems Science, 41(11), 1281 - 1291.

[6] Bournez, O. and Branicky, M. (2002). The mortality problem for matrices of low dimensions. Theory of Computing Systems, 35(4), 433-448.

[7] Courtois, N., Klimov, A., Patarin, J., and Shamir, A. (2000). Efficient algorithms for solving overdefined systems of multivariate polynomial equations. In Advances in Cryptology - EUROCRYPT 2000, International Conference on the Theory and Application of Cryptographic Techniques, Bruges, Belgium, May 14-18, 2000, Proceeding, pages 392-407.

[8] Daemen, J. and K., P. (2005). The self-synchronizing stream cipher mosquito: estream documentation, version 2. Technical report, e-Stream Project. Available at: www.ecrypt.eu.org/stream/p3ciphers/mosquito/mosquito.pdf.

[9] Daemen, J. and Kitsos, P. (2005a). The self-synchronizing stream cipher mosquito: estream documentation, version 2. eSTREAM, ECRYPT Stream Cipher Project, Report 2005/018. Available online at http://www.ecrypt.eu.org/stream.

[10] Daemen, J. and Kitsos, P. (2005b). The self-synchronizing stream cipher moustique. eSTREAM, ECRYPT Stream Cipher Project. Available online at http://www.ecrypt.eu.org/stream.

[11] Daemen, J. and Kitsos, P. (2008). The self-synchronizing stream cipher moustique. In New Stream Cipher Designs - The eSTREAM Finalists, pages 210-223. Springer-Verlag Berlin Heidelberg.

[12] Daemen, J., Govaerts, R., and Vandewalle, J. (1992). On the design of high speed self-synchronizing stream ciphers. In Proc. of the ICCS/ISITA'92 conference, volume 1, pages 279-283, Singapore. 
[13] Dravie, B., Guillot, P., and Millérioux, G. (2017). Design of selfsynchronizing stream ciphers: A new control-theoretical paradigm. In IFAC World Congress, (IFAC 2017), Toulouse, France.

[14] Dubi, C. (2009). An algorithmic approach to simultaneous triangularization. Linear Algebra and its Applications, 430(11-12), 2975 - 2981.

[15] Fliess, M., Levine, J., Martin, P., and Rouchon, P. (1995). Flatness and defect of non-linear systems: introductory theory and examples. Int. Jour. of Control, 61(6), 1327-1361.

[16] Hawkes, P., Paddon, M., Rose, G. G., and Miriam, W. V. (2004). Primitive specification for sss. Technical report, e-Stream Project. Available at: http://www.ecrypt.eu.org/stream/ciphers/sss/sss.pdf.

[17] Hellman, M. E. (1980). A cryptanalytic time-memory trade-off. IEEE Trans. Information Theory, 26(4), 401-406.

[18] Kasper, E., Rijmen, V., E.Bjorstad, Rechberger, C., Robshaw, M., and Sekar, G. (2004). Correlated keystreams in moustique. Technical report, ESTREAM Project.

[19] Maurer, U. M. (1991). New approaches to the design of self-synchronizing stream cipher. Advance in Cryptography, In Proc. Eurocrypt '91, Lecture Notes in Computer Science, pages 548-471.

[20] Menezes, A. J., Oorschot, P. C., and Vanstone, S. A. (1996). Handbook of Applied Cryptography. CRC Press.

[21] Millérioux, G. and Boukhobza, T. (2015). Characterization of flat outputs for LPV discrete-time systems: a graph-oriented approach. In 54th Conference on Decision and Control (CDC 2015), Osaka, Japan.

[22] Molland, H. and Helleseth, T. (2006). Linear properties in t-functions. IEEE Trans. Information Theory, 52(11), 5151-5157.

[23] Paterson, M. S. (1970). Unsolvability in $3 \times 3$ matrices. Studies in Applied Mathematics, 49(1), 105-107.

[24] Sarkar, P. (2003). Hiji-bij-bij: A new stream cipher with a selfsynchronizing mode of operation. IACR Cryptology ePrint Archive, 2003, 14.

[25] Sira-Ramirez, H. and Agrawal, S. K. (2004). Differentially Flat Systems. Marcel Dekker, New York.

[26] Strogatz, S. H. (2003). SYNC: The Emerging Science of Spontaneous Order. Penguin Group. 